\title{
Materials for Test Objects to Configure Aviation Optoelectronic Earth Remote Sensing Systems
}

\author{
E.B. Bablyuk ${ }^{1}, Y u . M$. Berlad $^{1}, A . G$. Letyago $^{2}$ and A.P. Kondratov ${ }^{1, *}$ \\ ${ }^{1}$ Moscow Polytechnic University, 107023 Moscow, Russia \\ ${ }^{2}$ Moscow Scientific-Research Television Institute, 105094 Moscow, Russia
}

\begin{abstract}
The paper illustrates the possibility of manufacturing deployable test objects to configure aviation optical equipment for remote sensing of the Earth in the form of a set of fragments made of weatherproof polymeric materials using the method of digital inkjet printing of two types of prints. The main fragments with a fixed optical density include images of light bars against a dark background. Background fragments are monochrome. We have suggested a reasonable combination of materials for test objects fragments, and assessed their optical parameters and lightfastness.
\end{abstract}

\section{Introduction}

Analogous optoelectronic systems for monitoring of the Earth's surface (MES) are being replaced by MES digital optoelectronic systems (OES). Unlike film systems, digital OES uses matrices of elementary photosensitive elements with a strictly ordered regular structure as an optical emission receiver (OER). Used as an optical emission receiver of digital OES, matrix (linear) charge-coupled photosensitive devices (CCPD) or metal-oxide-semiconductor complementary structures (MOSCS) have a fixed the physical size of an OER element. For this reason, if we want to assess the resolution power and to configure digital optoelectronic MES systems, it is necessary to create and to use bar test objects with a certain angular orientation of strips (bars) with respect to the regular structure of the optical emission receiver.

As the key MES OES performance evaluation criteria, the linear ground resolution of the onboard system $L_{M}$ is used.

The linear ground resolution is determined using the following formula:

$$
L_{M}=\frac{H}{2 R f}
$$

where $R$ - is the system's resolution power, $\mathrm{mm}^{-1} ; \mathrm{H}$ is the height of the aerial survey, $\mathrm{m}$; $\mathrm{f}$ is the system's focal distance, $\mathrm{mm}$.

The resolution power is a spatial-frequency characteristic of a photographic system. It is determined by visual decoding after photographing the test objects. The resolution power of MES OES is determined both in laboratory and in flight environments during navigation tests.

Configuration kits for airfield-based aviation optoelectronic systems include test objects containing calibrated photometric fields [1]. The spatial resolution of aviation equipment is assessed using bar test objects. Radiometric properties are assessed using photometric fields. There are two types of test objects: fixed objects manufactured by applying paint on a concrete base (the paint composition includes additives to ensure the required surface diffuseness) and deployable objects banners made of fabric or other flexible material with a printed pattern.

Test objects are rather material-intensive and shall have stable measuring properties throughout their service life. Their reasonable designing, manufacturing, deployment and maintenance of the surface in working condition shall meet high standards $[1+]$. Not only the choice of material for a test object is important, but also the technological process of printing the bars and bright fields, to ensure a high accuracy and stability of the pattern over large areas of the printed material.

The purpose of this study is justifying the optimum design, the choice of materials and the assessment of the bars and bright fields printing quality on a deployable test objects used to configure aviation optoelectronic system for remote sensing of the Earth.

\section{Research Objects and Methods}

To investigate the prints' optical properties, we selected banner materials of the "BlockOut" trademark, with a PVC coating.

Surface reflection spectral coefficients were measured using a "FieldSpec 3" spectroradiometer. The reflection coefficients were determined on a blank banner material before printing and on bright fields and bar elements with a variable tone intensity $(30 \%, 55 \%$ and $100 \%$ ).

Before each measurement, we determined the optical parameters of each white master object. The position

* Corresponding author: apkrezerv@,mail.ru

(C) The Authors, published by EDP Sciences. This is an open access article distributed under the terms of the Creative Commons Attribution License 4.0 (http://creativecommons.org/licenses/by/4.0/). 
height of the spectroradiometer's fiber optic cable lens was $15 \mathrm{~cm}$.

To assess the lightfastness, we used a print on the "Blockout" S-PRW334240B banner material.

The lightfastness was assessed by measuring the optical density of printed areas with a tone intensity of $55 \%$ and $100 \%$ after exposure of samples in a device according to the patent [6].

\section{Results and Discussion}

The properties analysis of weatherproof printed materials, that are available on the world market [5-7], allows us to conclude that banner fabrics with a PVC coating are suitable to manufacture test objects for remote sensing of the Earth. Synthetic fabric banners are

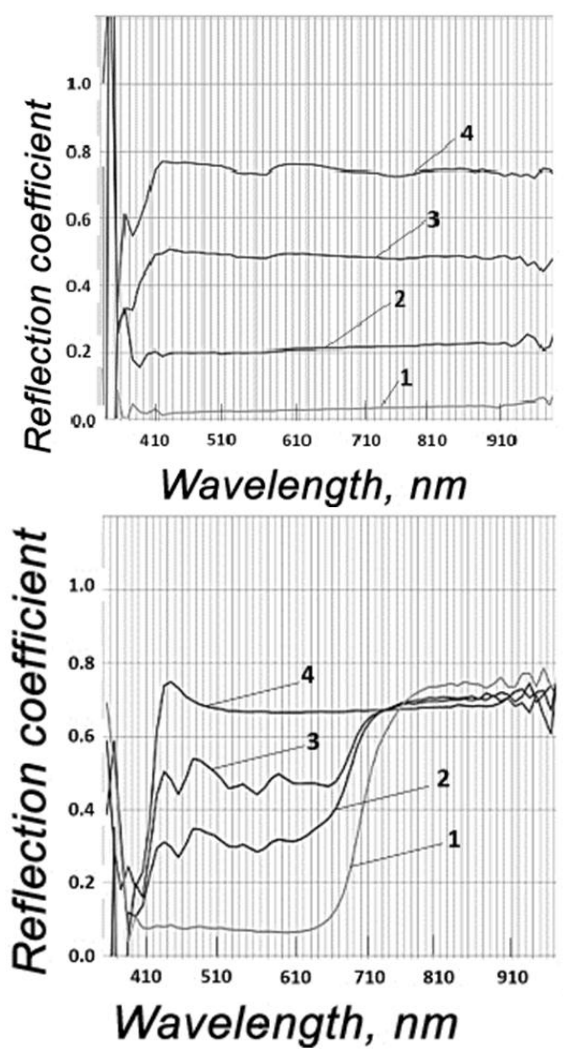

Fig. 1. The spectrum of light reflection coefficients from the prints on the fabric sample № 1 (a) and the fabric sample № 2 (b) with the tone intensity of $100 \%-1,55 \%-2$, $33 \%-3,1 \%-4$.

laminated composite materials that consist of a polyester mesh and a monolithic polyvinyl coating, intended for printing on polygraphic equipment. In terms of banners manufacturing technology, the mesh is filled with a solution or pasty composition (plastisol), containing polyvinyl chloride, and then it is passed through hot press rolls for imparting surface smoothness to the material, as required for printing.

The printed material for test objects shall have lightfastness, the minimum light transmittance and uniform electromagnetic radiation reflection characteristics in the visible spectrum before and after printing patterns (bars) with variable optical density thereon.

Based on a comparative evaluation of the physical and mechanical properties values, as declared by the manufacturer [7], and specific quality characteristics of the printed surface for manufacturing test objects and subsequent tests, we selected the following fabric banners: "BackLit" GLK S5251, "BlockOut" GLK S6151, "BlockOut" S-PRW334240B, "BlockOut" GLK $\mathrm{S} 5355 \mathrm{M}$, as well as and nonwoven fabrics made of synthetic fibers.

The values of the spectral reflection coefficients are presented in the form of charts (Fig. 1).

The light reflection coefficient from the non-printed fabric banner № 1 (1\%) within the range of 450 $1,000 \mathrm{~nm}$ has a constant value. The spectrum of light reflection coefficients from the prints with a tone intensity of $30 \%$ and $55 \%$ within the range of $400-600$ $\mathrm{nm}$ is unstable. All prints with a tone intensity of $(30 \%$, $55 \%$, and $100 \%$ ), that were obtained on the fabric banner sample № 2 (Fig. 1, b), have a constant value of light reflection coefficient in the wavelength range of 450 $1,000 \mathrm{~nm}$.

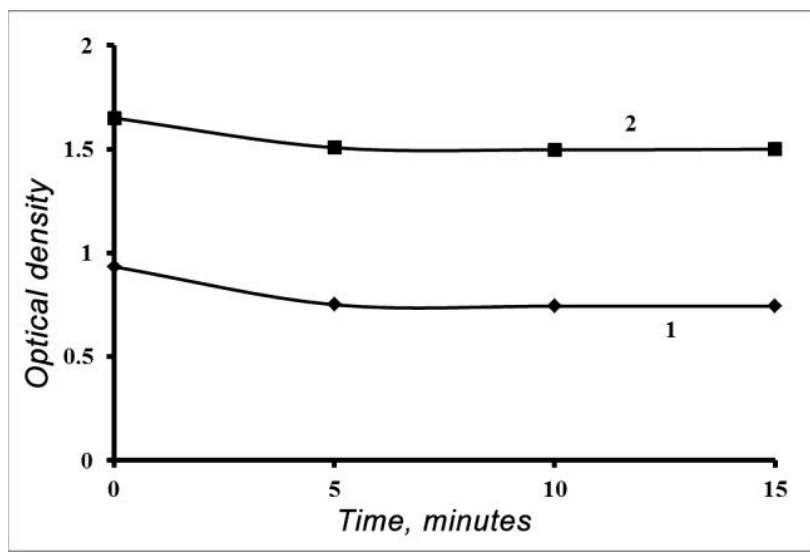

Fig. 2. Changes in the optical density of ink layer on different materials after printing using an UV ink: 1 - nonwoven material; 2 - banner fabric.

Modern banner inks are UV-curable, i.e. lightabsorbing compositions that significantly change their state of aggregation and physical properties when exposed to short-wave electromagnetic radiation. Therefore, it is reasonable to assume that during manufacture, immediately after printing and during a long-term use of test objects, the stability of their optical properties is likely to be affected not only by ultraviolet radiation, but also by daytime sunlight.

It has been found that the optical density of prints depends on the type of the non-printed fabric and varies significantly during 5 minutes (Fig. 2), whereas the color difference of the prints, as obtained on the banner fabric, exceeds the sensitivity threshold of human eyes, i.e. it is can be observed visually.

For experimental evaluation of lightfastness, we studied a print made on the "Blockout" S-PRW334240B banner fabric. 
The lightfastness was evaluated by measuring the optical density of printed areas with a tone intensity of $55 \%$ and $100 \%$ after aging the samples in a climatic chamber that simulated an accelerated deterioration of the material under the action of sunlight [6].

The testing results are presented in Table 1 . The test object according to the technical solution [7] consists of fragments with an image of bars of a certain group and unified lateral background fragments of the same geometric dimensions. The fragments are made of a banner fabric using a printing method that ensures the required optical measurement accuracy.

Table 1. Optical density values of the prints on the "Blockout" S-PRW334240B banner material before and after exposure to $\mathrm{UV}$ radiation

\begin{tabular}{|l|l|l|l|l|l|l|l|}
\hline $\begin{array}{l}\text { Exposure } \\
\text { time, hour }\end{array}$ & \multicolumn{3}{|c|}{0} & \multicolumn{3}{c|}{96} & \multicolumn{2}{c|}{180} \\
\hline $\begin{array}{l}\text { Tone } \\
\text { intensity, } \\
\%\end{array}$ & 55 & 100 & 55 & 100 & 55 & 100 \\
\hline $\begin{array}{l}\text { Optical } \\
\text { density }\end{array}$ & 0.848 & 1.226 & 0.852 & 1.228 & 0.846 & 1.224 \\
\hline
\end{tabular}

No less important task, besides increasing the accuracy of the measurements, shall be reducing the costs of consumables for manufacture of test object, saving time and labor costs as required for modules deployment to test specific operation modes of onboard optoelectronic equipment. The design of test objects provides for laying a set of modules, optimal for each type of the configured optoelectronic systems on the Earth's surface, consisting of one or more main fragments with a width that is the multiple of three meters, with groups of light bars against a dark background.

In determining the optimum set, it is necessary to take into account that the total width of the fragments formed in a module should exceed the width of the bar group deployed with respect to the long side of the module at an angle of $45^{\circ}$ or $135^{\circ}$. The number of auxiliary unified lateral background fragments, that are adjacent to the main fragments, should ensure at least two-fold excess of the distance from the bar pattern to the module edge.

The auxiliary background fragments, having similar characteristics (the same spectral reflection coefficient) against the background on the main fragments with bars, may be made of a nonwoven material that is less expensive, as compared to the banner fabric of the main fragments.

The evaluation of manufacturing costs for the existing [8] and assumed test objects is based on the comparison of the consumption of materials and related costs for manufacturing background fragments for one test object module with the same type of bars.

When manufacturing a test object, the manufacture of background fragments would need a printed material with a three-layer structure (including a reinforcing fabric and a lightfastness polymer coating) with a total surface area of 2.354 sq. m. When manufacturing background fragments of one test object module as per [7], a one-layer nonwoven material without coating is used, and the consumption of printed material for background fragments is 1.325 sq. $\mathrm{m}$. The saving of the printed material is up to $1.029 \mathrm{sq} . \mathrm{m}$, which is equivalent of $44 \%$ in terms of surface area, and significantly more in terms of cost.

The material's printing properties at background fragments differ substantially from those of banner fabrics with a PVC coating. The roughness of fabrics with a PVC coating is characterized by a value of 3-7 $\mu \mathrm{m}$. Due to the high surface strength and the optimum surface roughness of banner fabrics, the adhesion of printing ink layers reaches $70.0 \pm 0.9 \mathrm{MPa}$. The adhesive strength of the printing ink layers, that is used for printing the background of nonwoven fabrics, is only $17.0 \pm 0.8 \mathrm{MPa}$. However, this value is sufficient for multiple bending and folding the background fragment prints into a roll during their storage and unfolding on the Earth's surface.

\section{Conclusion}

We have demonstrated a possible manufacturing method by digital inkjet printing and presented a reasonable combination of materials for deployment of test objects to configure aviation optical equipment for remote sensing of the Earth.

\section{References}

1. F. Goryl, A. Burini, Calibration Test Sites and Cal/Val Portal Renovation. IVOS 21 (University of Lethbridge, Canada, 11/13 August 2009)

2. H. Kipphan. Handbook of Print Media (Springer, Moscow State Printing University, 2003)

3. S.A. Shadrina, A.V. Churkin, Polygraphy, 9, 17-20 (2014)

4. A.V. Churkin, Digital Printing. Key Methods and Equipment, In Proceedings of the All-Russian conference - Digital Printing. Issues of Technique and Technology, pp. 14-16 (2014)

5. A. Churkin, Polygraphy, 4, 39-40 (2015)

6. A.P. Kondratov, A Device to Determine the Chemical Stability and Lightfastness of the Ink Printing on a Polymeric Package, Patent of the Russian Federation № 153923, IPC G01N 17/02, (2016)

7. E.V. Altukhov, E.B. Bablyuk et al. A Test Pattern for Parameters Configuration and Determination of Optoelectronic Systems, Patent of the Russian Federation № 153923, IPC G01M 11/02 (2016)

8. Yu. G. Veselov, V. V. Tikhonichev, A. Danilin, Science and education, 4 (2012) 